\title{
Rabbit Retinal Ganglion Cell Activation Thresholds in Response to Various Electrical Stimulation Waveforms using a Different Spatial Resolution Electrode Array
}

\author{
M.E. ÇELIK ${ }^{a, *}$, M. ÖZDEN ${ }^{b}$, İ. KARAGÖZ ${ }^{a}$ AND G. SobACI ${ }^{c}$ \\ ${ }^{a}$ Electrical Electronics Engineering Department, Faculty of Engineering, Gazi University, Ankara, Turkey \\ ${ }^{b}$ Electrical Electronics Engineering Department, Faculty of Engineering, Kirikkale University, Kirikkale, Turkey \\ ${ }^{c}$ Department of Ophthalmology, Faculty of Medicine, Hacettepe University, Ankara, Turkey
}

\section{(Received May 26, 2016)}

\begin{abstract}
The analysis of retinal ganglion cell responses to electrical stimulation is invaluable way to understand how the retina reacts and develops. Artificial visual prosthetics have made progress to some extent. Although there are several studies about retinal ganglion cell responses in the literature, experimental findings and information about responses to special stimulation patterns for developing high-resolution visual prosthetics are quite limited. In this study, rabbit retinal tissue was stimulated by biphasic, monophasic and ramp function current pulses with various amplitudes and pulse widths in a set of in vitro experiments. Rabbit retinal ganglion cell responses and spike activities are comparatively analyzed based on the applied electrical stimulation parameters. We conclude that biphasic current pulses provide a lower stimulation threshold of $10 \mu \mathrm{A}$ with shorter pulse widths than those obtained by monophasic stimulation. Additionally, despite the fact that ramp function stimulation patterns have remarkable potential for eliciting spike formation with low stimulation thresholds, biphasic stimulation is chargebalanced and more convenient for high-resolution visual prosthesis systems.
\end{abstract}

DOI: $10.12693 /$ APhysPolA.131.1479

PACS/topics: 87.85.-d, 87.85.D-, 87.85.E-, 87.85.eg, 87.55.dh, 87.19.1b, 87.85.Ng

\section{Introduction}

Electrical stimulation is used clinically for the treatment of diseases such as epilepsy, for deep brain stimulation for Parkinson's disease, in cochlear implants, and for alleviating pain. Recently, electrical stimulation has been used to restore vision in blind patients. Vision is initiated by the light in a healthy eye. The photoreceptor layer, the outer layer of the retina, receives the light and transforms it into electrical signals that are delivered to the inner layers. Electrical signals are sent to the retinal ganglion cell layer followed by spatial and temporal processing and coding in the inner layers. The output from each retinal ganglion cell represents individual nerve fibers. Together, the fibers form the optic nerve, which conveys processed signals to the brain. If there is any damage to the visual pathway, the light entering the eye does not create any vision in the brain because of signal loss.

Vision may be lost because of certain diseases. Most such diseases are degenerative eye diseases such as agerelated macular degeneration (AMD) and retinitis pigmentosa (RP). These diseases primarily impair the photoreceptor layer of the retina. Although photoreceptors are damaged, most of the nerve cells along the visual pathway, especially retinal ganglion cells, remain intact, even in patients with advanced-stage disease [1-3]. Thus,

*corresponding author; e-mail: mahmutemincelik@gazi.edu.tr visual restoration could be provided by eliciting spots of light, called phosphenes, when remaining nerve cells, bypassing damaged parts, are electrically stimulated. A visual prosthesis could provide enough signal to enable basic mobility and navigation and provide the ability to sense surroundings in totally blind patients. Multidisciplinary studies have shown that retinal prosthetics could have potential for treating blindness caused by certain diseases that lead to photoreceptor degeneration.

Retinal prosthetics can be classified into 3 main classes depending on the anatomical region in which the implant is placed: epiretinal, subretinal, and suprachoroidal. Suprachoroidal prosthetics are placed in the region between the sclera and the choroid. Phosphene perception has been reported clinically for these prosthetics [4]. Although implantation is easier in terms of surgery and there is less risk of retinal detachment, these prosthetics do not provide the same spatial resolution that can be achieved by other approaches, and they require higher threshold currents to stimulate the retina because of the relatively long distance between the retina and the sclera [5-7]. With subretinal implants, electrodes are placed between the photoreceptor layer and retinal pigment epithelium layer. Chronic and acute trials demonstrated that reading large letters and object detection could be successively achieved [8-11]. There are some advantages, including advanced electrode fixation and short electroderetina distance, but subretinal implants are more risky in terms of surgery because of the delicate tissue. Retinal detachment is a risk, and there is limited space for implant and electronics [11-14]. The epiretinal approach is 
based on electrical stimulation of retinal ganglion cells, which mostly remain intact. There are several disadvantages to this method, including distorted stimulation patterns because of stimulation of axons near the electrodes, requiring more sophisticated image processing techniques to stabilize the implant in inner layers, but the advantage of the method is that implantation is easier and there is more available space in the vitreous cavity, which helps to dissipate the heat generated by the electronics [19]. Additionally, processed signals use the natural pathway in the retinal circuitry. These implants have the longest follow-up information and findings from animal experiments and acute and chronic human trials. Clinical studies performed with human subjects showed that recognizing and distinguishing object counters and reading letters with advanced correctness are possible [16-20].

In recent years, significant progress in the field of visual prosthetics has been made, with the contributions from different fields including biomedical engineering, electronics and medicine. Visual prosthetics that seek to restore visual perception are based on electrical stimulation of functional nerve cells that remain intact; the stimulation simply bypasses the damaged parts of the retina. One of the most important aspects of visual restoration is determining the optimal stimulation parameters because neural activity is controlled by electrical stimulation. Electrical stimulation is applied via a microelectrode array placed in the target tissue of the visual pathway. Epiretinal prosthetics are one of the most studied approaches to visual prosthesis systems.

Understanding the retinal response to electrical stimulation is critical for retinal prosthesis design and development. The limits of stimulation current pulses should be sufficient to excite the retinal ganglion cells with the lowest amplitude. These limits are dependent on how much the nerve cells have been damaged, the amplitude and frequency of the stimulation pulses, the dimensions and layout of electrode array (circular, square, hexagonal) and the status of electrode firing. Studies that used biphasic, monophasic stimulation patterns and fixed spatial resolution electrode arrays demonstrated that stimulation thresholds vary by experiment, and there is not a golden standard to determine the best conditions $[15,21,22]$.

In contrast to traditional stimulation patterns and approaches, using a hexagonal electrode array, which has dynamic spatial resolution and combinational stimulation pulses, enables an understanding of retinal response that is lacking in the literature. In this study, the responses of rabbit retinal tissues are considered, and responses are recorded under the same conditions for electrical stimulation but with different patterns. The stimulation patterns used in the in vitro experiments with rabbits were biphasic, monophasic and ramp functions, which had various stimulation amplitudes $(10,50,80,120 \mu \mathrm{A})$ and pulse widths $(100,200 \mu \mathrm{s})$. Spike activity was analyzed, and threshold currents were determined.

\section{Materials and methods}

This section includes three important procedures to be followed during the experiments: retinal preparation and interfacing with the electrodes, electrical stimulation and recording, and analysis of neural activity.

\subsection{Retinal preparation and interfacing with electrodes}

The Gulhane Military Medical Academy (GMMA) Animal Experiments Ethics Board approved the care and use of animals. Dark adapted female New Zealand rabbits weighing $2-3 \mathrm{~kg}$ were used. The rabbits were first sedated with intramuscular injections of ketamine $(40 \mathrm{mg} / \mathrm{kg})$ and xylazine $(10 \mathrm{mg} / \mathrm{kg})$. They were sacrificed by high dose intravenous sodium pentobarbital. After enucleation of the eyeball, the front part of the eye and vitreous were removed. The eyeball was segmented in $4 \mathrm{~mm}$ by $4 \mathrm{~mm}$ patches and placed in the Ames medium (Sigma Chemical Co.). The time between sacrifice and placement in the medium was approximately 4-9 min. The Ames medium was buffered with sodium bicarbonate and equilibrated with a gas mixture of $95 \%$ oxygen and $5 \%$ carbon dioxide. The retinal tissue was isolated by removing the sclera, retinal pigment epithelium, and inner limiting membranes with tweezers. The retina segment and a small amount of medium were placed in the microelectrode array chamber so that the retinal ganglion cells faced the electrodes. The medium was taken removed so that retina could adhere well to the electrodes. The temperature of the solution was set to $37^{\circ} \mathrm{C}$, and the chamber was perfused to feed the retina and enable long-term recording without any loss of signal. These steps were performed under red dim light. Standard perfusate (solutes in mM: $100 \mathrm{NaCl}, 30 \mathrm{NaHCO}_{3}$, 50 glucose, $6 \mathrm{KCl}, 2 \mathrm{MgSO}_{4}, 1 \mathrm{CaCl}_{2}, 1 \mathrm{NaHPO}_{4}$ ) was bubbled with $95 \% \mathrm{O}_{2}, 5 \% \mathrm{CO}_{2}$ with a $\mathrm{pH}$ of $7.5 \pm 0.2$ and a temperature of $37 \pm 1^{\circ} \mathrm{C}$. A system block diagram is presented in Fig. 1.

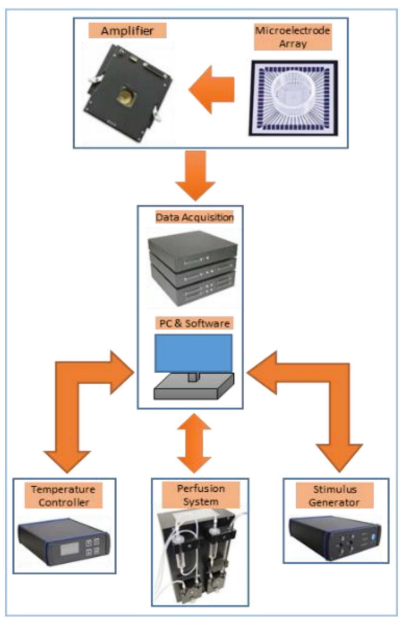

Fig. 1. Block diagram of experimental setup consisting of microelectrode array, amplifier, stimulus generator, perfusion system, temperature controller system and data acquisition module with PC. 


\subsection{Electrical stimulation and recording}

The recording system included several main components: a microelectrode array (60HexaMEA-Ti, Multi Channel Systems GmbH, Germany), an amplifier (MEA1060-Amplifier, Multi Channel Systems GmbH, Germany), data acquisition equipment and software, a stimulus generator (STG4002, Multi Channel Systems GmbH, Germany), a temperature controller and a peristaltic perfusion system. The microelectrode array used for the in vitro experiments had 60 channels and a hexagonal layout. The electrode dimensions and interelectrode distances were 10,20 , or $30 \mathrm{\mu m}$ and 30,60 , or $90 \mu \mathrm{m}$, respectively, as shown in Fig. 2. The specific layout is an idealized representation of the architecture of the retina. The density of neurons is more important in the center than in the periphery. This is matched by the density of electrodes on the MEA, which are also denser in the center than in the periphery. The electrodes in the center had a diameter of $10 \mu \mathrm{m}$, with an inter-electrode distance of $20 \mu \mathrm{m}$, and the peripheral electrodes had a diameter of $20 \mu \mathrm{m}$ and inter-electrode distance of $30 \mu \mathrm{m}$.

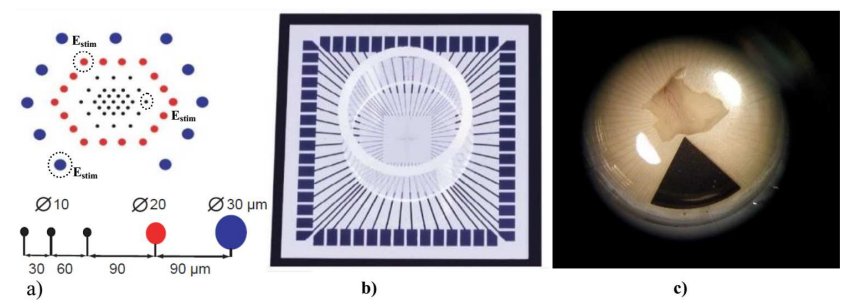

Fig. 2. (a) Schematic diagram. (b) Photograph of hexagonal electrode matrix used in experiments. (c) Fixed retina patch on hexagonal electrode matrix. Stimulation electrodes $\left(E_{\text {stim }}\right)$ are shown in (a).

The amplifier had a gain of 1000 and bandwidth of 1 $5000 \mathrm{~Hz}$, and it received information from 60 electrodes, each of which could be used for stimulation or recording, separately. Additionally, during the recording process, to prevent distortions caused by various sources, a Faraday cage and dark environment were used to isolate the system. An artifact appeared right after electrical stimulation. The artifact was quite different from others in terms of period and amplitude. The sampling frequency of the recording system was configured to be $25 \mathrm{kHz}$.

Stimulation trains with different amplitudes and pulse widths were formed. The amplitudes of the spike trains were $10,50,80$, and $120 \mu \mathrm{A}$, and the pulse widths were 100 and $200 \mu$ s. Biphasic, monophasic and ramp functions were used as stimulation patterns, as shown in Fig. 3. The stimulation current trains produced by the stimulus generator were delivered to selected electrodes of the hexagonal microelectrode array, and some electrodes were used as recording electrodes.

There were 20 successive stimulations that were applied for each stimulation type, including four different amplitudes and two different pulse widths. This procedure was repeated to obtain comparable results until the tissue died. Some stimulations were excluded because

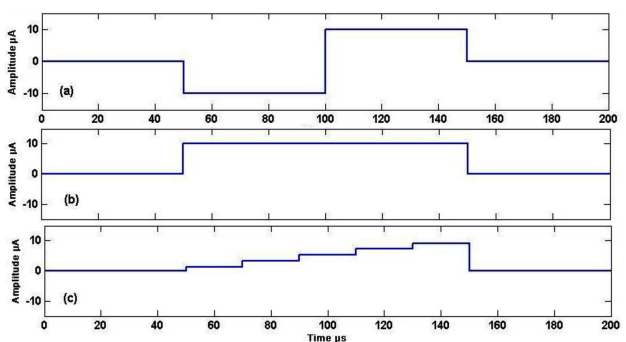

Fig. 3. Stimulation current patterns. (a) Biphasic stimulation. (b) Monophasic stimulation. (c) Ramp function.

of large amounts of signal distortion. There were 263, 129, 152 stimulation trials for biphasic, monophasic and ramp function stimulation, respectively. It was taken into consideration that there is a need for sufficient trials to compare the results for each stimulation types. Although we made preparations to provide complete contact with the retina by cleaning carefully and using a poly-lysine coating, the adhesiveness of the retina on the electrodes varied, which is a problem that has also been noted in the literature.

\subsection{Analysis of neural activity}

Following the electrical stimulation, neural activity was recorded. Raw neural activity and isolated spikes are shown in Fig. 4. Raw neural waveforms were transferred to the MATLAB environment and analyzed with custom scripts that included filtering with a $300 \mathrm{~Hz}$ highpass filter. Details are presented in another study [20].

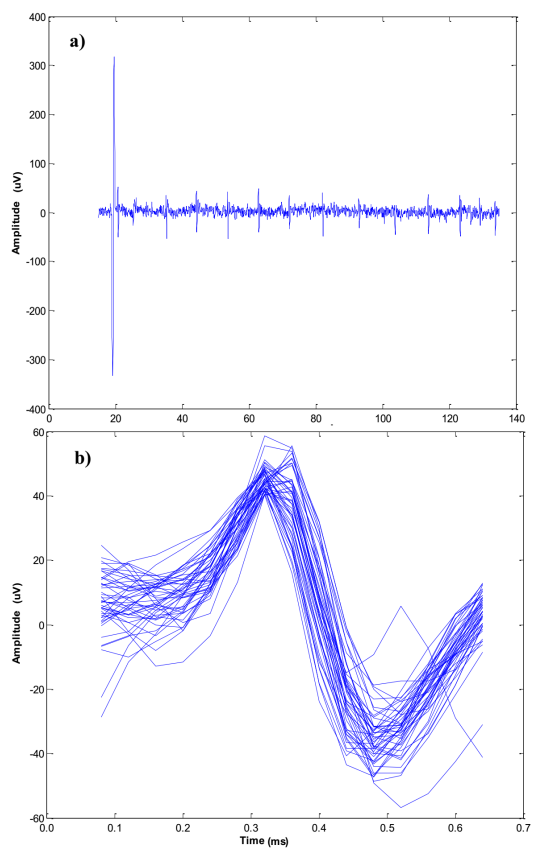

Fig. 4. Analysis of neural activity. (a) Raw neural waveform which is recorded and transferred to MATLAB environment. (b) Isolated spikes with multiple samples overlaid in order to use further steps. The $x$ and $y$ axes are in units of $\mathrm{ms}$ and $\mu \mathrm{V}$. 
Analysis of neural activity requires identifying and removing spontaneous activity. In this study, when the retina was in place and recording was started, the signal was only recorded until stability was reached, which was at least $10 \mathrm{~s}$. Then, electrical stimulations began. The stimulation process was completed in such a way that at least $10 \mathrm{~s}$ were recorded in a blank, unstimulated area, when the stimulation amplitudes changed. In the analysis process, to eliminate the misleading effects of spontaneous activity, the unstimulated areas were examined, and average spike counts were determined and used as the "background activity". Then, the spike counts were determined for gaps between consecutive stimulation pulses. The spike counts and background activity were compared. If the spike count observed between two successive stimulation pulses was greater than background activity, it was accepted that the first stimulation pulse generated the spikes. This procedure was repeated for each stimulation pulse with different amplitudes. A perfusion system was used to keep the cells alive. However, to prevent misleading effects that would create a difference between the beginning and end of the recorded signal, background analysis was performed based on the areas unstimulated for $10 \mathrm{~s}$, which were left for each stimulation amplitude. In this way, misleading effects of spontaneous activity were eliminated. The stimulation threshold was defined as the current amplitude that produced spikes with the highest percentage in repetitive stimulation pulses applied with a $2 \mathrm{~s}$ interval. Stimulation threshold success rate (STSR) was calculated for the most active electrode channels (MAECs) between $0 \%$ and $100 \%$ depending on how many stimuli excited spikes for applied consecutive pulses. Spike numbers and current thresholds were evaluated for the electrodes that showed the best recording in terms of signal to noise ratio. Whether the retina adhered the electrode or not had a huge impact on the recording performance. Recordings from all electrodes were not always available.

\section{Results}

Following the analysis of neuronal activity recorded after electrical stimulation, the results were presented by selecting some of the most active electrode channels (MAECs). Depending on the STSRs of the MAECs, the effects of biphasic and monophasic stimulation patterns on stimulation thresholds are shown in Figs. 5 and 6, respectively.

Neural activity is properly recorded. When the neural activity was analyzed, the stimulation threshold was determined to be $10 \mu \mathrm{A}$. The STSR for a $10 \mu \mathrm{A}$ stimulation pulse was determined to be $75 \%$. For 4 MAECs, success rates that elicited spikes for repetitive trials ranged from $22 \%$ to $100 \%$. Additionally, when pulse widths were examined, we found that stimulation trials with a pulse width of $100 \mu$ s gave a better result because they induced more spikes than the other pulse width, $200 \mu \mathrm{s}$. Pulses with $100 \mu \mathrm{s}$ and $200 \mathrm{\mu s}$ pulse widths elicited spikes at success rates of $93 \%$ and $29 \%$, respectively. Spike

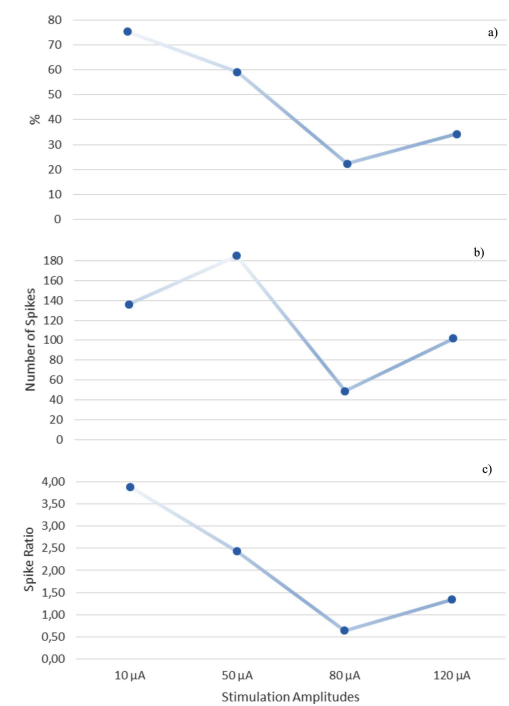

Fig. 5. Analysis results for biphasic stimulation patterns. (a) Stimulation threshold success ratio. (b) Spike count per pulse. (c) Spike ratio per pulse.

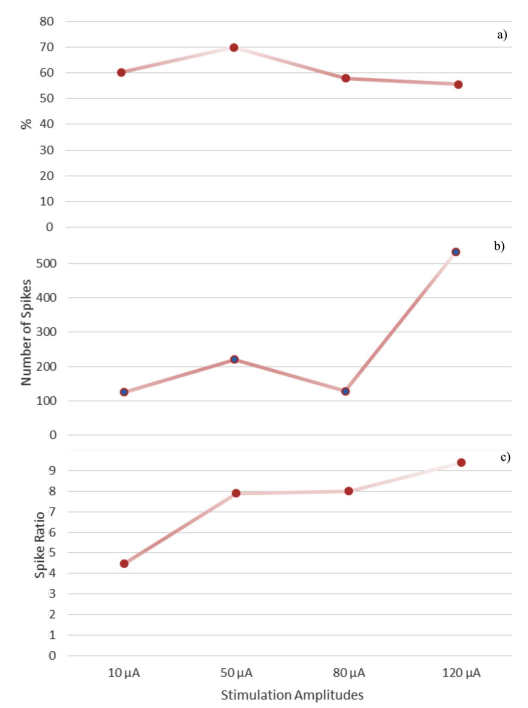

Fig. 6. Analyzed retinal responses to monophasic stimulation patterns. (a) Stimulation threshold success ratio. (b) Spike count per pulse. (c) Spike ratio per pulse.

count characteristics show that, as the stimulation amplitude increases, spike count and spike ratio per pulse increase, as reported in the literature (Fig. 5b). Following $10 \mu \mathrm{A}$ stimulation, spike count increased for a stimulation amplitude of $50 \mu \mathrm{A}$.

Applied monophasic stimulation showed that the current threshold needed to excite the retina is $50 \mu \mathrm{A}$, Fig. 6a. According to the 2 MAECs, success rates vary from $56 \%$ to $84 \%$. With regard to the effect of pulse width, longer pulses provide the minimum current threshold. Stimulation for 100 us provides a spike success rate of $57 \%$; stimulations with longer pulses of $200 \mu$ s have a success rate of $65 \%$. Additionally, spike count per pulse showed an increasing trend depending on the stimulation 
amplitude, as shown in Fig. 6b. Successive stimulation trials show that higher amplitudes generate more spikes. The number of spikes per pulse rises from $10 \mu \mathrm{A}$ to $120 \mu \mathrm{A}$ amplitudes.

Stimulation using a ramp function showed that the current threshold is $10 \mu \mathrm{A}$ for a pulse width of $100 \mu \mathrm{s}$. The averaged STSR was calculated to be $88 \%$. The stimulation elicited 582 spikes are detected by using 152 stimulation trials and it corresponds to average of 3.8 spikes per pulse. 8 MAECs showed that success rates changes between $10 \%$ and $100 \%$. Certain results, including the second part of stimulation series, could not be obtained. There were no significant findings, possibly because of the large distance between the retina and the electrode, which increases the electrode impedance.

Electrodes that showed spike activity after electrical stimulation were marked, and their results were analyzed. Electrodes that were used as stimulation electrodes are indicated in Fig. 2a; other electrodes were used for recording. These electrodes were used at different phases of analysis depending on the conditions under which they were recorded. The "activity ratio", AR, was determined for these electrodes individually. It was defined as the number of stimulation trials that generated spikes (positive trials) in one electrode divided by all positive trials in its category. The results are shown in Table I. For biphasic stimulation, the data from four electrodes were used. Three of them had diameters of $20 \mu \mathrm{m}$; the other had a $30 \mu \mathrm{m}$ diameter. The average AR of the $20 \mu \mathrm{m}$ diameter electrodes was $27 \%$, and it was $20 \%$ for the $30 \mu \mathrm{m}$ diameter electrode. In monophasic stimulation, two of the $10 \mu \mathrm{m}$ electrodes and two of the $30 \mu \mathrm{m}$ electrodes could be analyzed. The average AR of values of the $10 \mu \mathrm{m}$ and $30 \mu \mathrm{m}$ electrodes were $18 \%$ and $16 \%$, respectively. The AR of the $20 \mu \mathrm{m}$ electrode was $49 \%$. For ramp function stimulation, two $20 \mu \mathrm{m}$ electrodes and two $30 \mu \mathrm{m}$ electrodes were observed. Average ARs were calculated as $12 \%$ and $38 \%$, respectively. Based on the results, it was concluded that smaller electrodes are more effective on a large scale.

TABLE I

Comparison the effect of electrode sizes on spike activity.

\begin{tabular}{c|c|c|c|c|c|c|c|c|c|c|c|c|c}
\hline \hline Stimulation & \multicolumn{3}{|c|}{ biphasic } & \multicolumn{3}{c|}{ monophasic } & \multicolumn{3}{c}{ ramp function } \\
\hline channel & A1 & A2 & A3 & A4 & B1 & B2 & B3 & B4 & B5 & C1 & C2 & C3 & C4 \\
diameter $[\mu \mathrm{m}]$ & 20 & 20 & 20 & 30 & 10 & 10 & 20 & 30 & 30 & 20 & 20 & 30 & 30 \\
activity ratio [\%] & 23 & 30 & 28 & 20 & 15 & 5 & 49 & 18 & 13 & 14 & 10 & 13 & 63
\end{tabular}

\section{Discussion}

Safe stimulation limits and electrical stimulation parameters for neural prosthetic devices should be taken into consideration in the design and development systems for long-term use without removal. Safe stimulation, which means that electrical stimulation is applied within predefined limits, guarantees the creation of action potentials in retinal ganglion cells outputs without causing any damage or side effects. Charge injection limits, which depend on the electrode material, should be tolerable limits that avoid electrode corrosion and tissue damage.
Electrical stimulation plays an important role in neural activity. High-resolution systems for artificial sight restoration require stimulating the retina with the lowest possible stimulation current that can elicit distinguishable phosphenes. Thus, the determination of optimal stimulation parameters is quite important for effective stimulation.

The stimulation current thresholds are determined based on comparative analysis of the recordings, which are obtained using different stimulation patterns. Trials include a wide range of stimulation amplitudes. In vitro experiments serve as a basis for more detailed studies that can determine more accurate threshold levels. The number of MAECs changes in different cases, and may affect the analysis. This is because the electrode-tissue distance changes, and adherence of the tissue to the electrode may vary.

Optimal stimulation parameters must be determined to activate the retinal ganglion cells with the lowest amount of current possible. The optimal levels depend on how damaged the layers are, the stimulation amplitude, the pulse width, and the frequency of applied pulses. Additionally, the region of electrode placement, electrode size, inter-electrode distance and whether or not all electrodes are fired simultaneously have important effects on the optimal limits. Thus, the threshold limits reported in the literature vary. This work provides important findings about understanding retinal responses to electrical stimulation, and it includes different stimulation patterns, which is often lacking in the literature.

Biphasic and monophasic pulses were analyzed, which are two types of stimulation patterns that are frequently used in neural prosthetic devices. Biphasic stimulation was able to elicit spikes using lower threshold currents $(10 \mu \mathrm{A})$ than monophasic stimulation. Obtaining this result using short pulse widths of 100 ps also shows that short pulses are more suitable for direct stimulation of retinal ganglion cells. Shorter pulses directly activate the ganglion cells by triggering voltage-controlled sodium channels in the cell membrane. Hence, axons are the best places for stimulation because of the dense channels in the proximal fields of axons. Because it is thought that voltage-controlled sodium channels can be activated by rapidly changing charges, short stimulation pulses are ideal. For monophasic stimulation, a higher threshold level, $50 \mu \mathrm{A}$, was obtained. Pulse widths of 100 and 200 us had equal effects on current threshold. This shows that longer pulse widths elicit action potentials by using presynaptic neurons. Compared to retinal ganglion cells, photoreceptors and bipolar cells have different biological structures, and longer pulse widths could be more convenient to activate their ion channels [22, 23]. Less frequent stimulation pulses can activate the bipolar and photoreceptor cell network with lower threshold values than required for the activation of axons. When the ramp function was used, the stimulation threshold was determined to be $10 \mu \mathrm{A}$, and the pulse width was $100 \mu \mathrm{s}$. Because the ramp function has step-by-step increasing 
amplitude levels, it could be more successful in providing low threshold levels, but more detailed studies are required. Rapidly changing short pulses show better results for direct stimulation of retinal ganglion cells. Moreover, only ramp-based stimulation provided sampling of stimulation currents as steps. For some situations, interpretable results could not be obtained because of reduced retina-electrode contact or high electrode impedance. Spike counts per pulse (SCPP) were higher than for other types of stimulation, at the points at which stimulation thresholds were recorded. Additionally, spike counts per pulse decreased as threshold success ratio decreased. Regarding the effect of electrode dimensions on spike activity, smaller electrodes were more effective. For each situation, different electrodes were used because different stimulation points were used. This was because of better adhesion and close distance to active nerve cells.

\section{Conclusion}

Although visual prostheses are promising and provide hope for visually impaired people, clinical experiments performed with human subjects show only improved mobility and object localization. Pixelized vision, in which light points are provided by separate electrodes, has not been achieved. It is reported that vision including details of faces and objects is not possible because of the fading of light spots and other visual degradation. The current state-of-the-art, which includes low quality and inconsistency between patients, is far from ideal. The most important challenge to overcome is to provide higher resolution systems. To achieve this goal, the number of stimulation electrodes should be increased. An optimal electrical stimulation strategy should enable safe stimulation by forming distinguishable light spots. Using lower stimulation current within safe limits makes it possible to run more electrodes and to design high-resolution systems, and developing these systems requires determining the optimal stimulation patterns. This work shows that the biphasic stimulation method results in lower threshold levels by using shorter stimulation pulses, and it is more convenient for stimulating retinal ganglion cells.

\section{Acknowledgments}

This work is supported by Turkish Scientific and Technological Research Council (TUBITAK), Project Code: 113E181. Ethical statement: the Gulhane Military Medical Academy (GMMA) Animal Experiments Ethics Board approved the care and use of animals. It means that animal investigations are conducted according to the principles expressed in the Declaration of Helsinki.

\section{References}

[1] M.S. Humayun, J.D. Weiland, G.Y. Fujii, R. Greenberg, R. Williamson, J. Little, B. Mech, V. Cimmarusti, G. Van Boemel, G. Dagnelie, Vision Res. 43, 2573 (2003).

[2] D.Y. Yanai, J.D. Weiland, M. Mahadevappa, R.J. Greenberg, I. Fine, M.S. Humayun, Am. J. Ophthalmol. 143, 820 (2007).
[3] M.S. Humayun, J.D. Dorn, L. Cruz, G. Dagnelie, J.-A. Sahel, P.E. Stanga, A.V. Cideciyan, J.L. Duncan, Ophthalmology 119, 779 (2012).

[4] T. Fujikado, T. Morimoto, H. Kanda, S. Kusaka, K. Nakauchi, M. Ozawa, K. Matsushita, H. Sakaguchi, Y. Ikuno, M. Kamei, Y. Tano, Graefe's Arch. Clin. Exp. Ophthalmol. 245, 1411 (2007).

[5] K. Nakauchi, T. Fujikado, H. Kanda, T. Morimoto, Jun S. Choi, Y. Ikuno, H. Sakaguchi, M. Kamei, M. Ohji, T. Yagi, S. Nishimura, H. Sawai, Y. Fukuda, Y. Tano, Graefe's Arch. Clin. Exp. Ophthalmol. 243, 169 (2005).

[6] H. Kanda, T. Morimoto, T. Fujikado, Y. Tano, Y. Fukuda, H. Sawai, Investig. Ophthalmol. Vis. Sci. 45, 560 (2004).

[7] J.S. Brown, D.I. Flitcroft, G.S. Ying, E.L. Francis, G.F. Schmid, G.E. Quinn, R.A. Stone, Investig. Ophthalmol. Vis. Sci. 50, 5 (2009).

[8] E. Zrenner, K.U. Bartz-Schmidt, H. Benav, Proc. R. Soc. B Biol. Sci. 278, 1489 (2011).

[9] R.J. Jensen, J.F. Rizzo, Exp. Eye Res. 83, 367 (2006).

[10] F. Gekeler, P. Szurman, S. Grisanti, Graefe's Arch. Clin. Exp. Ophthalmol. 245, 230 (2007).

[11] S.R. Montezuma, J.I. Loewenstein, C. Scholz, J.F. III Rizzo, Investig. Ophthalmol. Vis. Sci. 47, 3514 (2006).

[12] D. Palanker, A. Vankov, P. Huie, S. Baccus, J. Neural Eng. 2, 105 (2005).

[13] A.Y. Chow, V.Y. Chow, Neurosci. Lett. 225, 13 (1997).

[14] K. Stingl, K.U. Bartz Schmidt, D. Besch, A. Braun, A. Bruckmann, F. Gekeler, U. Greppmaier, S. Hipp, G. Hörtdörfer, C. Kernstock, A. Koitschev, A. Kusnyerik, H. Sachs, A. Schatz, K.T. Stingl, T. Peters, B. Wilhelm, E. Zrenner, Proc. R. Soc. B Biol. Sci. 280, 1757 (2013).

[15] J.D. Weiland, M.S. Humayun, IEEE Trans. Biomed. Eng. 61, 1412 (2014).

[16] D. Yanai, J.D. Weiland, M. Manjunatha, R.J. Greenberg, I. Fine, M.S. Humayun, Am. J. Ophthalmol. 143, 820 (2007).

[17] T. Laube, C. Brockmann, G. Roessler, Graefe's Arch. Clin. Exp. Ophthalmol. 250, 51 (2012).

[18] M.S. Humayun, E. Jr. de Juan, J.D. Weiland, Vis. Res. 39, 2569 (1999).

[19] J.F. III Rizzo, J. Wyatt, J. Loewenstein, S. Kelly, D. Shire, Investig. Ophthalmol. Vis. Sci. 44, 5362 (2003).

[20] M.E. Celik, I. Karagoz, Acta Phys. Pol. A 128, B-297 (2015).

[21] A.P. Fornos, J. Sommerhalder, L. Da Cruz, J.A. Sahel, S. Mohand-Said, F. Hafezi, M. Pelizzone, Investig. Ophthalmol. Vis. Sci. 53, 2720 (2012).

[22] R.J. Jensen, O.R. Ziv, J.F. Rizzo, J. Neural Eng. 2, 16 (2006)

[23] C. Sekirnjak, P. Hottowy, A. Sher, W. Dabrowski, A.M. Litke, E.J. Chichilnisky, J. Neurophysiol. 95, 3311 (2006). 